\title{
Análisis de concordancia entre trazado cefalométrico manual y cefalométrico digital con programa Nemoceph.
}

\author{
Analysis of concordance between manual cephalometric and \\ digital cephalometric tracing with Nemoceph program.
}

\author{
Mariana Aguilar-Hernández, ${ }^{*}$ Israel de Alba-Cruz ${ }^{\ddagger}$
}

\section{RESUMEN}

La importancia del análisis cefalométrico dentro del diagnóstico en ortodoncia ha ido incrementando a través de los años, por ello, el interés de comparar la confiabilidad de los sistemas digitales con el trazado manual convencional. Objetivo: Definir el grado de concordancia entre los resultados de trazado cefalométrico manual y con Nemoceph. Material y métodos: Se utilizaron ocho medidas lineales y angulares del análisis cefalométrico de Steiner. Se realizó un estudio transversal, correlacional, en el cual se analizaron 70 radiografías laterales de cráneo digitales. Los resultados se dividieron en dos grupos, trazado manual y trazado cefalométrico con Nemoceph, los cuales fueron evaluados con un índice de correlación intraclase. Conclusión: Se reportó un grado de correlación intraclase mayor a 0.75 , estableciendo que el sistema digital exhibe la misma precisión del manual, con algunas ventajas convenientes a la época.

Palabras clave: Ortodoncia, cefalometría digital, radiografía.

\section{ABSTRACT}

The importance taken by the cephalometric analysis within the orthodontic diagnosis has been increasing over the years, for that reason the interest of comparing the reliability of the digital systems with the conventional manual tracing. Objective: To define the degree of concordance between the results of manual cephalometric tracing and with Nemoceph. Material and methods: Eight linear and angular measurements of Steiner's cephalometric analysis were used. A crosssectional, correlational study was conducted in which 70 digital skull lateral radiographs were analyzed. The results were divided into two groups; manual tracing and cephalometric tracing with Nemoceph, which were evaluated with an intraclass correlation index. Conclusion: a correlation degree greater than 0.75 was reported. Establishing that the digital system exhibits the same precision of the manual, with some advantages suited to the age.

Keywords: Orthodontics, digital cephalometric, radiography.

\section{INTRODUCCIÓN}

$\mathrm{E}^{n}$ n los últimos años, se han hecho grandes esfuerzos para desarrollar sistemas asistidos por computadora para la identificación de los puntos cefalométricos de referencia, ${ }^{1-3}$ debido a que los métodos digitales auxiliares para el diagnóstico presentan mayores ventajas, como almacenar de manera fácil y secuencial los estudios de los pacientes ortodóncicos. ${ }^{4}$ Sin embargo, desde 1989, cuando fue registrado el primer sistema digital intraoral para uso odontológico, ${ }^{5}$ se ha generado una discusión en torno al grado de concordancia de los resultados entre el sistema digital y el manual. En 1987, Savage realizó un estudio para comparar la localización de puntos en cefalometrías con operadores de diferentes grados de experiencia, y los resultados arrojaron que no influía la experiencia de los

* Cirujano dentista. Estudiante de la Maestría en Ortodoncia y Ortopedia Maxilar.

‡ Maestro en Ciencias de la Salud. Profesor de asignatura.

CINEO Instituto de educación y desarrollo profesional en Odontología.

Recibido: 04 Febrero 2019. Aceptado para publicación: 07 Septiembre 2020.

Citar como: Aguilar-Hernández M, de Alba-Cruz I. Análisis de concordancia entre trazado cefalométrico manual y cefalométrico digital con programa Nemoceph. Revista ADM 2020; 77 (5): 244-246. https://dx.doi.org/10.35366/96142 
operadores en la precisión de la localización de los puntos. ${ }^{6}$ En 1998, Stamm realizó una investigación donde comparó radiografías laterales convencionales con imágenes reproducidas en un monitor, y no encontró evidencia inequívoca de que algún método fuera mejor que otro. ${ }^{2}$ Por otro lado, Gregston en 2004 demostró que no hay diferencia en la confiabilidad del trazado manual con el uso de radiografías convencionales, ${ }^{7}$ radiografías convencionales digitalizadas por escaneado y radiografías digitales de placas de fósforo en la cefalometría de tejidos duros. ${ }^{8}$ En el 2005, Cohen describe la manera de cambiar o ajustar las dimensiones de la radiografía para obtener resultados más precisos a la hora de hacer sobreimposiciones digitales con radiografías de diferentes cefalostatos, así como la necesidad de calibrar la magnificación. ${ }^{9}$ De igual manera Roden-Johnson en el 2008 realizó un estudio para comparar la eficacia entre el trazado cefalométrico manual y con un programa digital, evaluando la localización de puntos de referencia, medidas lineales y angulares, y las sobreimposiciones que son aceptadas por la Junta Americana de Ortodoncia, no se encontraron diferencias significativas entre un método y otro en ninguno de los valores evaluados. ${ }^{10}$ También Tsorovas en 2010, en una evaluación de la eficacia de cinco programas digitales para cefalometría contra el análisis cefalométrico manual, llegó a la conclusión de que todos los programas y el trazado manual tienen la misma validez. La única ventaja que arrojó la investigación fue que el trazado cefalométrico manual toma el doble de tiempo que el digital. ${ }^{11}$ Según Segura en el año 2014, el programa Nemoceph $\mathrm{Nx}$ puede ser utilizado con toda fiabilidad en la realización de trazados cefalométricos. Dando como ventaja una disminución de tiempo, comparado con el trazado manual. ${ }^{12}$ Por último, en 2016 Farooq concluyó que el método digital es preferido sobre el método convencional en el uso diario gracias a sus ventajas, como la mejora, transmisión, almacenamiento y bajas dosis de radiación sin perder la calidad. ${ }^{13}$ Sin embargo, a pesar de las múltiples ventajas de los programas digitales, no existe un consenso sobre la mejor manera de realizar la migración del trazado manual al digital. Dado que el cambio a los métodos digitales es inminente, los profesionales de la ortodoncia deben estar preparados para que la transición se realice de la manera más segura posible. La comparación de los exámenes tradicionales con los que usan imágenes digitales es fundamental, con el fin de hacer esta transición.

\section{MATERIAL Y MÉTODOS}

Para la realización de esta investigación, se utilizó una muestra por conveniencia de 70 casos que asistieron al Departamento de Ortodoncia del Instituto CINEO durante el periodo escolar 2016 a 2018, los cuales contaban tanto con radiografía lateral digital como impresa. Los criterios de inclusión fueron las radiografías con dentición permanente completa, sin datos patológicos aparentes y sin distinción de sexo. Las radiografías digitales fueron tomadas con el aparato Orthophos XG plus bajo las siguientes condiciones: $73 \mathrm{Kv}, 15 \mathrm{~mA}$ y un tiempo de exposición de 9.4 segundos. Todos los trazos fueron realizados por el mismo operador. Los trazos manuales se midieron con la regla de Ricketts y los digitales con el programa Nemoceph. Los elementos angulares elegidos consistieron en los siguientes: SNA, SNB, ANB, ángulo interincisal. Las medidas lineales que se utilizaron fueron: distancia 1-NA, distancia 1-NB, segmento SE, segmento SL. El análisis estadístico se realizó en el programa SPSS versión 21, utilizando el índice de correlación intraclase para determinar el grado de concordancia.

\section{RESULTADOS}

En las 70 radiografías trazadas, se registró que el análisis de concordancia entre ambos métodos indica un $\mathrm{r}: \geq 0.75$, lo cual corresponde a la existencia de un alto grado de concordancia entre todos los parámetros utilizados de los dos métodos (Tabla 1).

\section{DISCUSIÓN}

El propósito de esta publicación fue evaluar la concordancia en las mediciones cefalométricas obtenidas de manera manual y mediante un programa digital. Los resultados obtenidos en este estudio reflejan que todos los parámetros

\begin{tabular}{lcc}
\multicolumn{3}{c}{ Tabla 1: Coeficiente de correlación intraclase. } \\
\hline Parámetro & ICC & IC 95\% \\
\hline SNA & 0.892 & $0.826-0.933$ \\
SNB & 0.915 & $0.863-0.947$ \\
ANB & 0.756 & $0.608-0.848$ \\
Ángulo interincisal & 0.750 & $0.596-0.843$ \\
1-NA & 0.884 & $0.813-0.928$ \\
1-NB & 0.936 & $0.896-0.960$ \\
SL & 0.782 & $0.593-0.876$ \\
SE & 0.868 & $0.786-0.918$ \\
\hline ICC = índice de correlación intraclase, IC = intervalos de confianza.
\end{tabular}


medidos tienen un coeficiente de correlación intraclase mayor a 0.75 , lo que significa una fuerza de concordancia excelente. Los resultados coinciden con lo registrado en la literatura internacional, donde diversos autores como Farooq, Chen y Tsorovas reportan que no existe diferencia significativa entre los resultados de ambos métodos. 11,13,14 El auge de la cefalometría digital se ve respaldada por sus múltiples ventajas como la fácil manipulación de la radiografía; el análisis digital nos proporciona una identificación de los puntos y estructuras anatómicas más rápida y precisa que el tradicional, y al utilizar radiografías en formato digital se reduce la contaminación, ya que elimina la necesidad de papel cefalométrico para efectuar el trazado. ${ }^{15,16}$ Otra de las ventajas es la baja exposición de radiación a la que se expone al paciente con estas radiografías y la facilidad de almacenamiento, ya que no consume espacio dentro de la oficina dental. Sin embargo, el hecho de necesitar un software especial involucra adquirir una licencia para el análisis cefalométrico digital, además de requerir una computadora con características especiales para soportar dicho software, situaciones que podrían significar desventajas para utilizar el método digital. ${ }^{14}$ Por otro lado, el análisis manual requiere una serie de equipo, como un negatoscopio, protractor, portaminas, entre otros, lo cual resulta poco práctico. Otra de las desventajas del sistema manual seria que el grosor de las líneas y puntos puede afectar el resultado al tratarse de mediciones milimétricas. ${ }^{15}$

\section{CONCLUSIÓN}

Gracias a los resultados obtenidos en esta investigación, donde se obtuvo un índice de correlación intraclase que no reporta diferencia significativa en las medidas realizadas con ambos métodos, podemos concluir que el sistema digital es una herramienta diagnóstica tan precisa como la manual y con mayores ventajas sobre ésta. Debido a la creciente actualización tecnológica y la inclusión de ésta dentro del Área de la Odontología, se facilita la adquisición de sistemas digitales auxiliares para la práctica clínica diaria, sugiriendo a la comunidad ortodóntica realizar la transición a los sistemas digitales a fin de unificar los criterios.

\section{BIBLIOGRAFÍA}

1. Forsyth DB, Davis DN. Assessment of an automated cephalometric analysis system. Eur J Orthod. 1996; 18 (5): 471-478.
2. Stamm T, Brinkhaus HA, Ehmer U, Meier N, Bollmann F. Computeraided automated landmarking of cephalograms. J Orofac Orthop. 1998; 59 (2): 73-81.

3. Rudolph DJ, Sinclair PM, Coggins JM. Automatic computerized radiographic identification of cephalometric landmarks. Am J Orthod Dentofacial Orthop. 1998; 113 (2): 173-179.

4. Rosero MK. Medios diagnósticos digitales en ortodoncia y cirugía ortognática. Revisión de Literatura. RevistaEstomatología. 2000; (5): 54-60.

5. Mouyen F, Benz C, Sonnabend E, Lodter JP. Presentation and physical evaluation of RadioVisioGraphy. Oral Surg Oral Med Oral Pathol. 1989; 68 (2): 238-242.

6. Savage AW, Showfety KJ, Yancey J. Repeated measures analysis of geometrically constructed and directly determined cephalometric points. Am J Orthod Dentofac Orthop. 1987; 91 (4): 295-299.

7. Geelen W, Wenzel A, Gotfredsen E, Kruger M, Hansson LG. Reproducibility of cephalometric landmarks on conventional film, hardcopy, and monitor-displayed images obtained by the storage phosphor technique. Eur J Orthod. 1998; 20 (3): 331340.

8. Gregston MD, Kula T, Hardman P, Glaros A, Kula K. A comparison of conventional and digital radiographic methods and cephalometric analysis software: I. Hard tissue. Semin Orthod. 2004; 10 (3): 204211.

9. Cohen JM. Comparing digital and conventional cephalometric radiographs. Am J Orthod Dentofac Orthop. 2005; 128 (2): 157-160.

10. Roden-Johnson D, English J, Gallerano R. Comparison of handtraced and computerized cephalograms: landmark identification, measurement, and superimposition accuracy. Am J Orthod Dentofac Orthop. 2008; 133 (4): 556-564.

11. Tsorovas G, Linder-Aronson Karsten A. A comparison of hand-tracing and cephalometric analysis computer programs with and without advanced features - Accuracy and time demands. Eur J Orthod. 2010; 32 (6): 721-728.

12. Segura FJE, Valverde AS, Ocampo AM, Angelares PRC. Estudio comparativo entre la cefalometría digital y manual con radiografías digitales. Rev Mex Ortod. 2014; 2 (2): 95-98.

13. Farooq MU. Assessing the Reliability of Digitalized Cephalometric Analysis in Comparison with Manual Cephalometric Analysis. J Clin Diagnostic Res. 2016; 10 (10): 20-24.

14. Prabhakar R, Rajakumar P, Saravanan R, Reddy A, Vikram Nr, Karthikeyan M. A hard tissue cephalometric comparative study between hand tracing and computerized tracing. J Pharm Bioallied Sci. 2014; 6 (5): 101.

15. Chen SK, Chen YJ, Yao CCJ, Chang HF. Enhanced speed and precision of measurement in a computer-assisted digital cephalometric analysis systemp. Angle Orthod. 2004; 74 (4): $501-$ 507.

16. Kochar GD, Jayan B, Chopra SS, Verma M, Kadu A, Singh S. Comparison of speed and precision of manual viz a viz computer assisted cephalometric measurements. J Pierre Fauchard Acad India Sect. $2015 ; 29$ (1): 11-20.

Correspondencia:

Mariana Aguilar-Hernández

E-mail: dra.mariana.aguilar@gmail.com 